Para enlazar con este artículo / To link to this article:

http://dx.doi.org/10.14198/fem.2017.29.03

Para citar este artículo / To cite this article:

Flores Hernández, Aurelia y Lorena Jiménez Montes. «Socialización y sociabilidad de género en un escenario universitario altamente masculinizado». En Marcos Jesús Iglesias Martínez e Inés Lozano Cabezas (coords.), La (in) visibilidad de las mujeres en la Educación Superior: retos y desafíos en la Academia. Feminismo/s, 29 (junio 2017): 71-98, DOI: 10.14198/fem.2017.29.03

\title{
SOCIALIZACIÓN Y SOCIABILIDAD DE GÉNERO EN UN ESCENARIO UNIVERSITARIO ALTAMENTE MASCULINIZADO
}

\author{
SOCIALIZATION AND SOCIABILITY OF GENDER IN \\ A MASCULINIZED UNIVERSITY CONTEXT
}

\author{
Aurelia FLORES HERNÁNDEZ \\ aure7011@hotmail.com \\ orcid.org/0000-0002-8465-7485 \\ Lorena JIMÉNEZ MONTES \\ anerolflor@hotmail.com \\ orcid.org/0000-0002-4227-1373
}

Secretaría de Educación Pública del estado de Tlaxcala (México)

\section{Resumen}

El propósito de este trabajo es explorar los desafíos y las tensiones que enfrentan estudiantes universitarias en las prácticas de sociabilidad y modos de convivencia a los que recurren para relacionarse con otros en la cotidianeidad de un espacio educativo construido y reconocido socialmente para el género masculino y donde la presencia de las mujeres se mimetiza. Para tal fin se recurre a la reflexión teórica sobre socialización diferencial de género y sociabilidad, adoptando a la antropología de la experiencia como un paradigma metodológico, mediante la aplicación directa de entrevistas. El estudio es de corte cualitativo. Los hallazgos sugieren que, en el sistema escolar universitario estudiado, las prácticas de sociabilidad y convivencia están mediatizadas por camuflajes de género, útiles y convenientes para la continuidad de un sistema de sexismo y violencia, que si bien nace antes de la Universidad, en ésta se consolida.

Palabras clave: género, sociabilidad, socialización, Universidad 


\begin{abstract}
The purpose of this article is to identify and to understand the challenges that university students face in the practices of sociability and codes of coexistence that they use as skills to interact with other people, in the daily life of a university context built socially for the masculine gender and, in which woman mimic the actions of those around them. We discuss the concepts of gender differential socialization and sociability, adopting the anthropology of experience like a methodological perspective, we use the application of interviews, and the study is qualitative. Findings suggest that in the university school system these practices of socialization and codes of coexistence are mediated by gender camouflage, useful and convenient for the continuity of a sexist and violent system that, although born before university, is remarkably resilient.
\end{abstract}

Keywords: gender, sociability, socialization, university 


\section{INTRODUCCIÓN}

En la última década, se ha observado un creciente aumento de estudios que documentan los avances en el conocimiento sobre las variadas circunstancias que las mujeres de modo aguerrido desafían para ingresar, permanecer, egresar y laborar en disciplinas fuertemente masculinizadas. En el contexto de este estudio, en este artículo se discute la clara ausencia en la reflexión de este tema en la Universidad Autónoma de Tlaxcala. Aún más, a nivel institucional, el camino hacia procesos de igualdad es muy corto, en particular en escenarios educativos de alta incidencia masculina como la Facultad de Ciencias Básicas Ingeniería y Tecnología (FCBIyT). Con este trabajo se pretende explorar los desafíos y las tensiones que enfrentan estudiantes universitarias en las prácticas de sociabilidad y modos de convivencia a las que recurren para relacionarse con otros en la cotidianeidad de un espacio educativo construido y reconocido socialmente para el género masculino y donde la presencia de las mujeres se mimetiza.

Inicialmente se debaten teóricamente dos conceptos fundamentales, la socialización diferencial de género y la sociabilidad. Además se distingue la influencia de la socialización y las prácticas de sociabilidad en las relaciones de género en la Universidad. Para tal efecto, se expone un apartado que aborda las investigaciones efectuadas en contextos educativos universitarios de alta concentración masculina en varios países de Latinoamérica. La revisión se hizo con el fin de reconocer ciertas peculiaridades del ingreso y tránsito de las mujeres en estos espacios, así como el impacto de estas prácticas entre diversos agentes universitarios. Los frentes que a las mujeres les toca sortear tanto en las interacciones como en los mecanismos institucionales indican una férrea y casi inamovible estructura universitaria de fuerte arraigo patriarcal.

En la segunda sección se aclara el procedimiento metodológico, el cual se centra en el enfoque de la antropología de la experiencia que coloca el interés en las experiencias de las participantes, organizadas mediante narrativas, vía la aplicación de técnicas cualitativas (entrevista temática y observación). El estudio fue aplicado en una universidad pública mexicana, ubicada en la región centro de este país. Como característica de este organismo universitario de cuatro décadas de formación, se observa un escenario formativo y laboral 
de alta segregación del género femenino en campos tradicionales e incipiente presencia de políticas institucionales pro-igualdad.

A la luz de las experiencias narradas por las estudiantes en tres espacios (físicos, las aulas y simbólicos), en los resultados se estructuran los principales hallazgos en el contorno de dos categorías analíticas: sociabilidad y convivencia con el estudiantado, y sociabilidad y convivencia con el profesorado.

A modo de conclusión se indica que a pesar de los costos de ingresar en seis disciplinas formativas (matemáticas aplicadas, química industrial, química, sistemas electrónicos, computación y mecatrónica), de los avatares que significa formarse y de los retos de ejercitar la profesión, las mujeres están y forman parte de nuevos profesionales en esos campos. No obstante, para transitar por los espacios universitarios, y ante la presencia de prácticas de sociabilidad y convivencia que ellas no sienten seguros y miran con desconfianza, ellas recurren a estrategias de resguardo para pasar desapercibidas y egresar, convencidas de que están en carreras de su elección porque cuentan con el intelecto para lograrlo.

\section{EL ENCUADRE TEÓRICO}

La socialización es un proceso mediante el cual el ser humano se ajusta a las formas y modo de vida de un grupo social en particular, es decir, el comportamiento humano debe adaptarse a las normas socioculturales de su hábitat. Peña y Buschiazzo (15), retomando la conceptualización de Rocher (1980), indican tres aspectos fundamentales de la socialización: la adquisición de la cultura, su internalización en la personalidad y la adaptación al entorno social. En este proceso de aprendizaje, los elementos socioculturales se recrean y las personas los matizan en sus propias vivencias a lo largo de toda su existencia.

La socialización primaria o principal permite al sujeto transformarse en un ser social y adquirir las pautas básicas esperadas para cada individuo en una sociedad instaurada. Los agentes socializadores centrales en esta dinámica son la familia, el grupo de pares, la escuela, la iglesia, el Estado y el mercado, entre otros, y funcionan como instituciones ideológicas, laborales o políticas responsables de este proceso. Sobre estas bases se instituye la socialización secundaria, en la cual las personas asumen como suyos determinados patrones -hábitos, comportamientos y normas- (Peña y Buschiazzo 17).

La socialización es un recurso útil para inculcar a hombres y mujeres los papeles o roles pre-asignados, apropiados en la infancia y reproducidos durante el ciclo vital. La familia (medio familiar) y la escuela (medio social) son dos de las instancias básicas de reproducción de prácticas socializadoras y en ellas se forjan códigos, valores, normas y expectativas socioculturalmente esperadas 
para cada género (Preciado et al 44). La socialización de género modela la feminidad y la masculinidad a partir de cánones establecidos según se nace hombre o mujer. En esta dinámica, los sujetos asumen funciones prescritas en los márgenes de una tradicional, rígida e histórica división sexual del trabajo. Lo culturalmente conveniente es que los hombres y las mujeres se adapten y adopten las normas sociales para formar parte del núcleo familiar y social que los resguarda.

En razón de que cada sociedad posee diferentes visiones de lo que es ser hombre o mujer, el valor asignado a cada género es flexible. Esta visión social varía según cultura, época histórica, espacio geográfico y otras categorías (edad, raza, estilo de vida, color de piel, la preferencia sexual, etcétera). Sin embargo en cada una se encuentra presente una constante: las mujeres son concebidas jerárquicamente inferiores y existe una distribución desigual de recompensas sociales valoradas con base a estereotipos de género, donde el proceso de socialización delimita el accionar del binomio masculino/femenino (Peña y Buschiazzo 18).

A través de la socialización diferencial, que está presente como un proceso inherente a la sociedad misma, se logra que los individuos adecuen su comportamiento y su identidad previamente normalizados y definidos a los modelos y a las expectativas creadas para los sujetos masculinos o femeninos. La socialización de género constituye un proceso de aprendizaje cultural de los papeles atribuidos a cada quien según su sexo. Las mujeres y los hombres en este aprendizaje reconocen los espacios que les corresponden de acuerdo a esquemas heteronormativos de género (Peña y Buschiazzo 17, Lorente 27).

En el sistema educativo, la escuela funciona como institución formadora y al mismo tiempo como sitio donde el sistema sexo/género es legitimado. En su papel de agente de socialización, refuerza los cimientos para la generación y la difusión de esquemas sexistas, prejuicios, estereotipos y representaciones sociales diferenciales (Larena y Molina 203).

La sociabilidad simboliza «el gusto por estar juntos». Las interacciones con los pares, el gozo de asociarse, de tener compañeros(as) y amigos(as), representa un aspecto lúdico de reunirse con otros (Simmel 95) y emotivo-afectivo que estimula a los sujetos a relacionarse e interaccionar con sus semejantes (Navarro 1994, citado en Pérez e Ibarra 4). Además de que en cada grupo se establecen normas y valores propios que implican la socialización entre pares (Weiss 1269). Pérez e Ibarra precisan:

La socialización es el proceso mediante el cual el sujeto se adapta a la estructura social [...] Por su parte la sociabilidad nos explica la forma en que 
interaccionamos o nos comunicamos entre sí en una sociedad determinada; es decir, las formas en que nos vamos haciendo individuos de esa sociedad concreta. Ambos conceptos socialización y sociabilidad nos ayudan a entender los estilos de sociedad o como se constituyen las sociedades (4).

De modo específico, la función de la escuela, no solo como replicadora de información/conocimiento, sino como espacio de intercambio de socialización entre sexos, reproductora de la identidad, de la sexualidad y de esquemas binarios de género, es puerta abierta a la perpetuación de la socialización diferenciada (Flores 74, Martínez y Solís 166). Empero, el contexto político, económico y social actual demanda alejarse de una escuela homogeneizadora y «reconocer que las desigualdades sociales atraviesan también las paredes de las escuela» (Malegarie y Tavernell 120). Por tanto, es urgente una educación basada en una socialización en igualdad que dignifique la vida humana (Cantillo y Rocha 2, Lorente 34).

En la escuela, como entidad de aprendizaje y de educación, se sostiene no sólo la desigualdad, sino también la violencia a través del recurso de la socialización (Lorente 27). De este modo, es permisible que durante el recreo las niñas permanezcan arrinconadas en lugares lejanos para protegerse y evitar pelotazos o golpes, mientras los niños ensanchan el uso de los espacios de juego (Costa y Rogério 4). De igual modo, las actividades deportivas que constituyen un espacio ilimitado de vida cultural al mismo tiempo visibilizan la discriminación hacia las mujeres (Gallego y Estebaranz 1). El ejercicio físico estimula el desarrollo social y ciudadano (perseverancia, equilibrio, liderazgo), y ciertos indicadores de sociabilidad (solidaridad o participación social). No obstante, la práctica deportiva es casi siempre menos frecuente entre las mujeres, en parte por el difícil acceso a las instalaciones deportivas (Gallego 1).

Empero, si bien los sistemas educacionales son funcionales a la socialización por la claridad y delimitación de su misión (Peña y Buschiazzo 26), no todos los espacios educativos son homogéneos, pues cada caso se encuentra regido por normas internas. En la escuela, las relaciones entre jóvenes se manifiestan en diferentes formas, interacciones grupales o intergrupales (y de inter e intra géneros) en ajuste casi siempre a las reglas de los centros educativos. En estas relaciones ocurren sucesos contradictorios, desde actos de discriminación, estigmas, exclusión y de violencia hasta la presencia de eventos solidarios, expresiones de reciprocidad afectiva positiva, responsabilidad e identidad grupal (Paulín et al 2). 


\subsection{La Universidad como espacio de continuidad de la socialización}

Los espacios de sociabilidad y su forma de apropiación son diferenciales según género, edad, condición social, entre otras categorías, aunque éstos serán definidos por la valoración otorgada social y culturalmente a lo femenino y a lo masculino. En todo caso, los sitios de sociabilidad también funcionan como espacios de socialización, la Universidad es uno de ellos. Esta institución educativa ha asumido un papel formativo integral donde la socialización de género se ha convertido en una extensión perene de prácticas de sociabilidad oscilantes entre sus contornos dentro y fuera. La escuela -y la Universidad- es un espacio de sociabilidad donde quienes se identifican en ella aprenden a relacionarse, a conocerse a sí mismos, colocan en acción su identidad, la redefinen o la asumen, al mismo tiempo que al contacto con otras identidades, la fortalecen (Rebolledo 90).

La Universidad custodia aún una sólida y férrea estructura jerárquica e ideológica que continúa alentando relaciones de desigualdad y, por supuesto, de socialización diferenciada de género (Larena y Molina 203, Cantillo y Rocha 1). Un trato diferenciado en función del sexo es un atentado contra la construcción de una vida digna; perjudica las posibilidades y potencialidades de las personas e invalida el desarrollo de ciertas capacidades. Esta diferenciación socializadora conforma actitudes, creencias y modos de relacionarse con los demás y el mundo (López et al 358).

Entre los aspectos que se han reflexionado en el binomio Universidad y violencia se encuentran precisamente las implicaciones diferenciales de la socialización de género, que supone comportamientos y creencias divergentes entre el estudiantado, enraizadas desde la familia, que conducen a prácticas sexistas, discriminación y violencia. El reconocimiento de la violencia de género como un problema sistémico que afecta de forma significativa la experiencia de las universitarias y atraviesa la convivencia cotidiana dentro de la Universidad se manifiesta en variadas aristas (Mingo y Moreno 144). En la cotidianeidad de la vida universitaria, la circulación de un ambiente adverso hacia las mujeres es un «pan de todos los días» que se ancla no sólo en la dinámica relacional entre grupos y sexos, sino en los programas de estudio, en los debates de las aulas y en la compleja «cultura institucional de género» (Mingo y Moreno 139, Larena y Molina 208).

En ese mundo cultural universitario, el ejercicio de la masculinidad tiene múltiples manifestaciones, en su mayoría cargadas de fuerte violencia (Maceira 202) y modos sutiles y simbólicos de expresión. Por ejemplo, el nombrado «dominio conversacional masculino», que permite la interrupción del turno de habla de las mujeres o el uso mayor de tiempo por parte de los hombres, 
constituye una forma de control de la voz y de los contenidos de las conversaciones (Martínez 27). De igual modo, esta superioridad de la palabra se traduce en mayor dedicación en cuanto a tiempo por parte del profesorado y con ello también orientación sobre el tema tratado, aunque se tiende a justificar que los hombres reciben mayor atención porque requieren más disciplina y necesitan controlar su comportamiento (Coates 189).

En otras investigaciones (Castro y Vázquez 612; Vázquez y Chávez 63) se ha reconocido que entre universitarios uno de los temas predilectos para murmurar son precisamente «las mujeres» (sexualidad, cuerpo, albures, apariencia física). Este tema se convierte en un elemento de identificación de ser hombre y un vínculo socializador entre varones. Ante el maltrato del que las mujeres padecen y son objeto, ellas manifiestan reacciones y sentimientos ambivalentes que se balancean entre aguantar, sufrir, callar, o bien protestar, rechazar y resistir (Camacho 141). García et al. (15) afirman que las universitarias prefieren relacionarse entre sí para compartir secretos, sentarse juntas en la clase y estudiar.

\subsubsection{Sociabilidad en escenarios formativos masculinizados}

Para las mujeres, transitar en campos educativos universitarios «celosamente masculinos» tan hostiles es un desafío que simula caminar en terrenos minados. La presencia de las mujeres en la Universidad -en particular en los campos con alta concentración de hombres- irrumpió los modelos y estereotipos de género hegemónicos (Preciado et al 43). Pero la escasa participación de las mujeres en las nombradas «ciencias duras» propició el encuentro de diversos estudios y corrientes reflexivas para su comprensión, específicamente, la escuela feminista se ha interesado en el orden de género prevaleciente en estos escenarios universitarios (Guevara y García 10, Mingo 28).

La inmersión de las mujeres en carreras «no convencionales» resulta de mecanismos sociales de selección y exclusión, algunos de ellos directamente asociados con el régimen de género y arraigado en el imaginario social (Arango 152). Esta situación las hace aparecer y figurar casi siempre como «invisibles o extranjeras» (Mingo 31), «recién llegadas» (Ballarín 36), «invitadas» (Peña y Buschiazzo 6), «intrusas» (Buquet et al 9) o «invasoras». Aunque algunos opinan que la palabra invasión está mal empleada, puesto que no consideran que ciertas profesiones sean privativas del género femenino. Lo que ocurre simplemente es que los hombres accedieron a un espacio que desde siempre estuvo allí y ahora las «mujeres han ocupado cada vez más espacio en la universidad, pero aún no la habitan» (Ballarín 19). Tal afirmación se hace evidente 
al mostrar los fuertes privilegios que mantienen los hombres, especialmente en áreas tradicionales e históricamente forjadas para ellos (Preciado et al 20).

Entre las mujeres, la decisión de ingresar en alguna ingeniería tiene respuestas ambivalentes. Por un lado, puede estar asociada a estímulos recibidos en el plano familiar (Arango 143) o por el contrario a la renuencia social para apoyar esta decisión (Álvarez et al 10). Al respecto, Oliveros et al (93) destacan que en la Ingeniería Aeroespacial de la Universidad Autónoma de Baja California, México, la motivación de la elección de esta carrera entre las mujeres se cimienta en decisiones informadas y responde a precedentes familiares, aunque en la mayoría de los casos ninguna mujer integrante de las familias de las estudiantes se representó como modelo femenino a seguir, lo que muestra los casi nulos ejemplos de mujeres exitosas en este campo del conocimiento.

Por su parte, Guevara y García encuentran en su estudio que, para las estudiantes entrevistadas, la elección de estudiar ciencias -exactas y naturales- se manifestó como un reto personal plagado de intereses, necesidades y deseos, búsqueda de reconocimiento, prestigio y desarrollo intelectual; así como «la opción por una identidad disciplinaria asociada a una actividad que no corresponde al mundo tradicional de las mujeres» (12). Las autoras refieren que en este contexto fueron fundamentales tanto el apoyo familiar (padres/madres) y el estímulo del profesorado como los logros individuales obtenidos por su potencial académico.

No obstante, según resultados del trabajo de investigación de Buquet et al. en la Universidad Nacional Autónoma de México (UNAM), las mujeres muestran un mayor logro en indicadores de rendimiento (por ejemplo, el avance en créditos) en el campo de las Ciencias Físico-Matemáticas e Ingenierías. Este resultado no favorece la contratación laboral femenina a futuro y ellas son quienes menos potencialidad tienen de obtener un empleo bien remunerado, pues prevalece un sistema de estereotipos que les atribuye menores capacidades para estas áreas y otorga mayor reconocimiento a los hombres como los más aptos.

También en esa máxima casa de estudios mexicana, Mingo explora el trato y las prácticas discriminatorias que las mujeres padecen. El estudio muestra los agravios hacia las estudiantes en un ambiente universitario adverso, hostil y desequilibrado, no sólo en cifras, sino en términos del discurso. Según la autora, las mujeres deben lidiar en el día a día con chistes sexistas, lenguaje soez, albures, murmullos, injurias, descalificaciones, hostilidad, risas sonoras, tensiones por excesiva carga académica, sensación de aislamiento y de encontrarse al margen, soledad, fatiga y malestar. Estas realidades representan «ejemplos de la energía extra que [ellas] han de invertir para batallar con los actos con los que se delimita la posesión masculina de un territorio» (33). 
Con la intención de indagar en la experiencia de mujeres en la educación profesional en dos facultades de Ingeniería-Mecánica y Electrónica, y Civil- de la Universidad de Colima, México, Preciado et al. (41) proponen un análisis en tres niveles donde se focaliza la opresión/resistencia: el género, la raza y la clase. Los autores matizan que en el contexto cultural del grupo se afianza y continua la reproducción de una «cultura de ingeniería masculina» y de violencia simbólica a través del lenguaje (comentarios y bromas machistas). Además, en estos dos espacios, las estudiantes vivencian discriminación de género por parte de profesores y de sus compañeros, frente a lo cual ellas aparentan ser uno más de ellos.

El camuflaje que significa «disfrazarse» es el método que permite a las estudiantes en contextos educativos altamente masculinizados «pasar de largo» e indistinguibles durante toda su formación. Las estudiantes adoptan esta estrategia para acomodarse en ambientes lacerantes machistas, pues les permite parecerse o asemejarse al resto de la comunidad universitaria, pasar desapercibidas. Para lograrlo, la vestimenta es central. Mingo (32) comenta que las jóvenes de su estudio limitan su forma de vestir para evitar «el chifladero», de modo que este hecho funciona como un efecto inhibidor.

Esta situación es muy similar a la que encuentran Peña y Buschiazzo (60 89 y 118) entre estudiantes de la ingeniería de la madera, donde de acuerdo a su opinión ellas enfrentan un medio adverso que las obliga a masculinizarse -vestir como hombre y actuar como tal-, pues es la alternativa para validarse en ese mundo. Asimismo, las estudiantes identifican dos mecanismos para reducir la discriminación en su contra. Primero, demostrar continuamente que son capaces de ocupar ese lugar, retando y compitiendo con sus congéneres para validar su presencia y confirmar su intelecto (inteligencia femenina); ante esta actitud, los hombres que se insertan en este tipo de carreras no tienen nada que aclarar. Segundo, representar actitudes de indiferencia frente al menosprecio de parte del profesorado que las coloca como poco aptas para la vida científica y muy hábiles para los quehaceres domésticos.

Otras disciplinas caracterizadas por mantener «un sello masculino», pero que aparentemente suponían en su origen mayor apertura para las mujeres (en razón de habilidades manuales y condición física) debido al manejo de innovaciones tecnológicas, que son distintas a carreras masculinas tradicionales, no lograron del todo la inclusión femenina de modo igualitario. Arango (152) afirma que la ingeniería en computación es una de las profesiones más apreciadas en la sociedad colombiana, cubre bajos porcentajes de participación femenina y una marcada preferencia de parte de las mujeres por involucrarse en áreas gerenciales, tarea que implica trato directo con usuarios, encierro en 
oficinas y acciones prácticas empresariales, y de menor cobertura en habilitación para el diseño de programas.

Estos escenarios masculinos que se consideran «más armónicos» para el tránsito de las mujeres durante sus estudios, las atribuyen al aprovechamiento de habilidades propias de su sexo. La experiencia de estudiantes en la carrera de arquitectura en Colombia indica que ellas acceden a esta profesión porque mantienen la idea de contar con dotes manuales, alta creatividad y visión de lo estético, herramientas que seguramente les ayudarán a diseñar ambientes agradables para el ser humano. La investigación también detecta que las relaciones entre mujeres y hombres son aisladas, distantes y de aguda competencia académica (Cantillo y Rocha 7).

En general, los resultados de las investigaciones revisadas sugieren que la socialización diferencial de género, que germina en la familia y en los primeros contactos relacionales, es vigorizada con la participación de cada uno de sus agentes en la Universidad. Sin embargo, ninguno de estos trabajos puede considerarse como concluyente. La realidad se transforma continuamente y, en ocasiones, un ambiente puede ser totalmente distinto a otro, aun cuando en apariencia sostengan las mismas características o perfiles. Por tanto, es posible decir que estos escenarios de socialización y sociabilidad son estrechos y «cerrados», y en ellos las mujeres deben permanentemente demostrar su capacidad, más allá de las virtudes «naturales» impuestas pues la circulación cotidiana de actos de subestimación, discriminación y violencia, que casi siempre, las obliga a recurrir a la mimetización como un mecanismo de resguardo y protección. Para contribuir a la reflexión de estas complejas aristas, este artículo se interesa por la comprensión de las prácticas de sociabilidad y modos de convivencia que las universitarias emprenden en un contexto escolar fuertemente masculinizado y, sobre todo, las contradicciones de la socialización diferenciada de género.

\section{LO METODOLÓGICO}

La antropología de la experiencia es una perspectiva que coloca a la agencia humana en la comprensión y construcción de toda vida social (Díaz 7). Esta plantea que las experiencias traducidas mediante las narrativas -como construcción social- se organizan y dan significado a la experiencia individual. Estas estructuras sirven como guía de interpretación, incluyen pensamientos, deseos, temores, sentimientos y disposiciones. Esta mirada antropológica convino como un punto de partida metodológico de gran apoyo para extraer las voces de las participantes. La proposición metodológica de este estudio se centra en el plano de las experiencias, reconociendo que éstas pueden ser flexibles y 
referirse a lo habitual o a lo reflexivo, pero siempre en perspectiva de lo subjetivo (Álvarez-Gayou 51). Se recurre a las interacciones y los significados a la luz de las narrativas -la palabra-y su interpretación de quienes son participes en la investigación.

El trabajo es de corte cualitativo, perspectiva favorable porque permite reconocer la capacidad de interpretar y significar las experiencias por las propias personas (Martínez y Solís 149). La particularidad de la metodología reside en que se centra en el estudio de los sujetos con la intención de reflexionar acerca de la situación vivida para comprender lo que ocurre. En específico, es de interés la identificación de las relaciones y los significados de la problemática estudiada sin importar la representación estadística de los hechos (Sánchez 118). Se utilizó una «muestra de conveniencia» (Hernández 145), cuya pretensión no es responder a criterios estadísticos ni buscar una representatividad del fenómeno estudiado o alcanzar la generalización de resultados.

\subsection{Instrumentos y recuperación de la información}

El plan de investigación se estructuró en tres etapas, la primera comprende el diseño del protocolo y la ubicación del contexto de estudio; la segunda corresponde a la elaboración de los instrumentos de recopilación de la información y el desarrollo del trabajo de campo; y la etapa final cubrió la sistematización, el análisis y la interpretación de la información.

Las actividades de trabajo de campo se desarrollaron en un periodo de seis meses (junio a noviembre del año 2015), plazo en el cual se participó activamente en distintos escenarios y eventos de la Facultad de Ciencias Básicas, Ingeniería y Tecnología. En esta etapa se cumplieron dos propósitos: (1) notas derivadas de observaciones in situ durante los dos primeros meses, aunque la técnica de observación se mantuvo a lo largo de todo el periodo y (2) detección de potenciales candidatas a participar y aplicación de entrevistas.

La selección de las participantes comprometía su colaboración voluntaria. Previa explicación de los propósitos del estudio, las entrevistas se aplicaron durante dos meses en un plazo intermedio, en horarios diversos y en sitios convenidos por las propias estudiantes, todos ubicados en el interior de la universidad. El total de participantes fue de diecisiete estudiantes inscritas en diferentes semestres de seis disciplinas formativas del campo de las ingenierías.

La entrevista utilizó un guion temático, incluyendo los siguientes ejes:

a. Datos generales de la entrevistada (edad, semestre cursando, carrera, sexo, procedencia)

b. Motivos de elección de la carrera y circunstancias presentes 
c. Experiencias de sociabilidad en espacios físicos de la facultad estudiada (biblioteca, oficinas administrativas, auditorio, sala de computación o laboratorios, cubículo de fotocopiado, sanitarios, estacionamientos, canchas deportivas, cafetería, áreas verdes y pasillos)

d. Experiencias de sociabilidad en las aulas (el salón de clases)

e. Experiencias de sociabilidad en espacios simbólicos (eventos universitarios como: homenajes -formar parte de la escolta, del grupo de edecanes o emitir un mensaje-, conferencias, talleres, actividades deportivas, graduaciones o fiestas juveniles institucionales, elecciones estudiantiles para representantes de grupo o formar comités o representaciones ante el consejo universitario)

Con la intención de reconocer las prácticas de interacción, modos de convivencia y sociabilidad entre el estudiantado y con el profesorado también se utilizaron las notas de campo en los tres escenarios. Las notas de campo se centraron en la identificación de anuncios, avisos en murales, grafiti, situaciones rutinarias, eventos, festejos, hechos significativos que se desarrollan en este centro universitario, diálogos formales e informales, gestos, tonos de voz, pausas, lenguaje corporal, volumen de la conversación, vestimenta, accesorios utilizados, colores, actitudes corporales, entre otros.

\subsection{Sistematización y análisis de la información}

Para el procesamiento y análisis de los datos cualitativos se retomó la propuesta de Mejía (48), quien sugiere hacer una lectura de las narrativas o de los textos y se efectúe una examinación en espiral de cada caso y del conjunto, donde se incluya la recuperación de información de las notas de campo, logrando un análisis detallado y profundo del tema tratado.

Después se hizo una lectura relacional siguiendo el orden de los apartados centrales de la entrevista. Con esta disposición se logró identificar características distintivas, lo que permitió distinguir similitudes y diferencias, las cuales son expuestas mediante las narrativas, eligiendo aquellas que denotaran la singularidad del conjunto de casos. Es importante decir que las experiencias de sociabilidad se distinguieron a través de las prácticas relacionales que las entrevistadas sostenían con el estudiantado y con el profesorado, razón por la cual la estructuración de los resultados retoma dos categorías analíticas expositivas: sociabilidad y convivencia con el estudiantado, y sociabilidad y convivencia con el profesorado a la luz de las experiencias en los espacios señalados.

El paradigma interpretativo, útil como filtro de la experiencia, apoyó la reflexión, pues centra su interés en la comprensión de las significaciones de las 
experiencias y prácticas en el ámbito escolar. A fin de recuperar la perspectiva de las estudiantes y «comprender el sentido de la acción en el marco de un nivel de análisis intersubjetivo», se reconoce que quien realiza la labor investigativa también contiene en sí subjetividades (Paulín et al 3).

\section{EL ESPACIO UNIVERSITARIO ESTUDIADO}

La Universidad Autónoma de Tlaxcala (UAT) se localiza en el estado de Tlaxcala, México. Se encuentra constituida en cuatro divisiones: 1) Ciencias Sociales y Administrativas; 2) Ciencias y Humanidades; 3) Ciencias Biológicas y 4) Ciencias Básicas, Ingeniería y Tecnología. De esta última forman parte las Facultades de Ciencias Básicas, Ingeniería y Tecnología; y Diseño, Arte y Arquitectura. El estudio fue aplicado en la Facultad de Ciencias Básicas, Ingeniería y Tecnología (FCBIyT), la cual hasta el año 2016 ofertaba seis licenciaturas: Ingeniería en Computación, Ingeniería Mecánica, Ingeniería Química, Sistemas Electrónicos, Matemáticas Aplicadas y Química Industrial.

Esta Facultad surgió en el año 1978 como Departamento de Ingeniería y Tecnología, orientado a diversas áreas de la ingeniería química (petroquímica, automotriz, alimentos, tratamientos de aguas, contaminación, farmacéuticos). De manera inicial, los planes de estudio se estructuraban en 10 semestres o cinco años escolares. En el último año se elegían las especialidades: Química Industrial e Ingeniería Química. En el año 1984 se aprobaron los planes de estudios de ambas licenciaturas y al siguiente año, 1985, se integran otras dos disciplinas: Ingeniería Mecánica e Ingeniería en Computación, y más tarde, en 1987, la Licenciatura en Matemáticas Aplicadas. A partir de este año se reconoció como Departamento de Ciencias Básicas, Ingeniería y Tecnología.

Durante la mitad de los noventa se incorporan dos posgrados (Maestría en Ingeniería Química, Ciencias de la Calidad y Ciencias en Ingeniería en Computación) y al final de la misma década se abrió la Ingeniería en Sistemas Electrónicos (1999). En el año 2005 se reconoció como Facultad de Ciencias Básicas, Ingeniería y Tecnología, y para el año 2009 se creó el Doctorado en Ciencias en Ingeniería Química http://ingenieria.uatx.mx/fcbiyt/vision/.

Como se observa en la tabla 1, la población estudiantil de la FCBIyT es de prevalencia masculina. La admisión porcentual de hombres ha rebasado en los últimos tres ciclos escolares el 66\%; sin embargo, la matrícula de mujeres ha ido en ascenso al menos un punto porcentual anual. En particular, las carreras de computación, química y química industrial tienen una mayor presencia femenina, el resto (sistemas electrónicos, mecánica, y matemáticas) son disciplinas masculinizadas. Esta distribución supone una polarización binaria de la ciencia, que atribuye socioculturalmente estas disciplinas a las ciencias duras 
que conllevan a la comprensión de dimensiones complejas, donde el orden femenino (apegado a la subjetividad) no tiene cabida.

Tabla 1. Matrícula -nivel ingeniería/licenciatura-

Facultad de Ciencias Básicas Ingeniería y Tecnología (2013-2016)

\begin{tabular}{|c|c|c|c|c|c|c|c|c|c|c|}
\hline \multirow{3}{*}{ Ingeniería } & \multicolumn{10}{|c|}{ Ciclo Escolar } \\
\hline & \multicolumn{3}{|c|}{$2015-2016$} & \multicolumn{4}{|c|}{ 2014-2015 } & \multicolumn{3}{|c|}{ 2013-2014 } \\
\hline & Hombres & Mujeres & Total & Hombres & & ijeres & Total & Hombres & Mujeres & Total \\
\hline Computación & 298 & 85 & 383 & 289 & & 99 & 388 & 330 & 112 & 442 \\
\hline $\begin{array}{l}\text { Sistemas } \\
\text { Electrónicos }\end{array}$ & 40 & 14 & 54 & 50 & & 6 & 56 & 61 & 6 & 67 \\
\hline Mecánica & 83 & 6 & 89 & 83 & & 4 & 87 & 77 & 5 & 82 \\
\hline Química & 72 & 83 & 155 & 59 & & 75 & 134 & 64 & 83 & 147 \\
\hline $\begin{array}{l}\text { Química } \\
\text { Industrial }\end{array}$ & 26 & 58 & 84 & 24 & & 42 & 66 & 19 & 31 & 50 \\
\hline $\begin{array}{l}\text { Matemáticas } \\
\text { aplicadas }\end{array}$ & 44 & 38 & 82 & 37 & & 35 & 72 & 40 & 34 & 74 \\
\hline Total & $56366.5 \%$ & $28433.5 \%$ & 847 & $54267.5 \%$ & 261 & $32.5 \%$ & 803 & $59168.5 \%$ & $27131.5 \%$ & 862 \\
\hline
\end{tabular}

Fuente: ANUIES

El campus universitario de la FCBIyT contiene grandes espacios, áreas verdes, deportivas y de esparcimiento (cafetería, cancha de básquetbol/voleibol y campo de fútbol) y zona académica (aulas, cubículos, talleres, laboratorios, centros de cómputo, biblioteca, auditorio, sanitarios, cubículo de fotocopiado) que se encuentran bien delimitadas y equipadas para el ejercicio de múltiples y diversas actividades. El estacionamiento es amplio y es frecuente encontrar letreros indicativos para ubicar las aulas de clase. El edificio académico es moderno con aulas amplias y equipadas para la impartición de diversas asignaturas. También cuenta con un edificio de tres salones grandes con iluminación que se utilizan para la impartición de cursos de posgrados y/o para la presentación de exámenes de grado.

\section{LOS HALLAZGOS EN LAS INGENIERÍAS ESTUDIADAS}

\section{1. ¿Por qué estudiar una ingeniería?: «es algo de lo que todos huyen»}

Uno de los primeros cuestionamientos que se exploró fue conocer las razones e intereses que las mujeres tuvieron para estudiar determinada carrera en la FCBIyT. Se encontraron diversas opiniones que indicaron que las ingenierías 
son un desafío, pues son áreas formativas consideradas racionales y que «elevan la inteligencia».

Las matemáticas es algo de lo que todos huyen, todos se espantan, entonces si es un reto para nosotras pues a pesar de que es complicado, nosotras tenemos una meta, terminar una carrera y a pesar de que sabemos que es difícil o que a veces nos cuesta pasar los exámenes, le echamos ganas y pues ya, al final de cuentas, sacamos la materia (Marly, estudiante de matemáticas)

A pesar de que esta disciplina es considerada como un campo severo y arduo tanto para hombres como para mujeres, en particular ellas refieren es necesario transitarlo como un camino pedregoso, por lo que lo asumen como un desafío que son capaces de superar. Otras opiniones aludieron que las disciplinas que cursan representan áreas innovadoras, novedosas e interesantes. Por ejemplo, la formación en computación permite «el uso de la tecnología» que en opinión de las entrevistadas es «la vanguardia de un mundo cambiante» que les abrirá nuevas expectativas de vida.

Si de igual forma porque me gusta lo que nos enseñan, es la carrera que tiene más amplio campo de trabajo y es muy versátil (Karina, estudiante de computación)

Si a mí me gusta por las materias que nos dan y el campo de trabajo (Ángeles, estudiante de computación)

Algunos puntos de vista de las estudiantes de la carrera de química insinuaron que si bien su disciplina de estudio es complicada, también goza de un campo laboral llamativo tanto profesional como económicamente:

Es precisa, muy interesante, difícil, el campo laboral es extenso y bien pagado (Manuela, estudiante de química)

La carrera exige mucho trabajo y está muy enfocada al campo de la investigación y eso es lo agradable (Mercedes, estudiante de ingeniería química)

Tiene su grado de importancia y su grado de dificultad y te puedes desenvolver en la docencia, la investigación o a nivel industrial (Karla, estudiante de química industrial)

La ingeniería química es un área de apertura laboral, debido al impacto del desarrollo de habilidades en la formación de recursos humanos, la investigación y la inmersión en empresas o laboratorios. Esta característica, según las entrevistadas, permite una continua y permanente capacitación, particularmente la investigación se presenta como un campo innovador de empleo.

Otras respuestas indicaron el interés de estudiar este tipo de carreras porque en el futuro podrán dedicarse a actividades de enseñanza. Esta perspectiva, si bien reproduce la práctica docente dentro de esquemas tradicionales de la 
división sexual del trabajo, también podría considerarse como una coyuntura para seguir abriendo oportunidades profesionales a otras mujeres en la actividad científica y dejar paso a la presencia de modelos femeninos que rompan el mito de «esto es cosa de hombres». No obstante, todavía algunas estudiantes en la Facultad visualizan ciertas limitaciones en las expectativas laborales: «A veces la mayoría de la gente cuando vas a pedir trabajo te dicen: 'No hay, las ingenierías son para los hombres'...» (Roberta, estudiante de mecánica). Estas experiencias las perfila y posiciona frente a tratos desiguales de género en el ejercicio de su futura carrera. Al respecto, dos estudiantes comentan lo que el profesorado les ha dicho:

La profe si nos ha dicho que a la hora de pedir trabajo si vamos a tener problemas (Roberta, estudiante de mecánica)

Nos han comentado que para una mujer es más difícil ser matemático por que llevan la casa, llevan a los niños y la parte académica, entonces es más complicado para una chica (Marly, estudiante de matemáticas)

Es muy posible que las dificultades a las que se refiera Roberta tengan que ver con obstáculos para un ejercicio profesional pleno (encontrar empleo, preferencias de hombres para las contrataciones, ubicaciones de los sitios de empleo, horarios, entre otros), pero también al conjunto de normas socioculturales que estigmatizan los papeles de género, tal como lo enunció Marly.

Del total de las entrevistadas, sólo una estudiante expresó su predilección hacia otras disciplinas de las ciencias sociales. El resto respondió estar bien convencida en el momento de elegir qué estudiar, entre ellas asumieron tener habilidades intelectuales para las carreras elegidas, particularmente el manejo de las matemáticas y del uso de la tecnología computacional. Sin embargo, como lo precisan, esta decisión de estudiar con «mayoría de hombres» ha tenido costos adversos, por ejemplo, sentirse incomodas y hostigadas por comentarios como «esa carrera es de niños», «seguramente porque estudias eso no le das importancia a tu arreglo personal»o «los matemáticos son un poquito fachositos».

Mediante la observación se notó que la vestimenta cómoda, a la moda y según la edad las identificaba como «universitarias», identidad caracterizada por usar habitualmente tenis o zapato bajo, pantalón de mezclilla, sudadera, escasamente ropa formal o zapato de tacón alto. Los accesorios entre ellas eran escasos, lo mismo el uso de maquillaje. Es posible que ésta sea la estrategia para pasar desapercibida y contribuya a esta personificación de imágenes. Incluso una estudiante indicó que no le gusta pasar por el patio grande cuando hay tanta gente y prefiere rodear el edificio para llegar al aula.

En el contexto estudiado, ocasionalmente algunas de ellas participan en eventos académicos como edecanes o en otras actividades escolares 
(presentaciones académicas, cívicas, eventos sociales, entre otros) visten de modo serio: «En la escuela cuando me piden que venga formal puedo tal vez utilizar un vestido» (Ana, estudiante de química); «Ahorita no venimos tan formales, hay lugares y momentos en los que tenemos que vestir bien, también en la manera de como hablamos, somos más correctos al hablar, ya no decimos tantas groserías» (Vero, estudiante de sistemas electrónicos).

De esta manera, las mujeres son encasilladas a «vestir bien» en tanto participen en eventos sociales o académicos dentro del campus universitario, pues como éstos son consideradas actividades oficiales ellas pueden portar vestimentas que de modo habitual no usarían por el riesgo a sufrir burlas o enfrentar situaciones incomodas.

\subsection{Sociabilidad y convivencia entre el estudiantado: «sacan temas que incomodan»}

La convivencia grupal es diversa y de un promedio de siete $\mathrm{u}$ ocho personas, se encuentran grupos de varones donde dos a tres mujeres conversan o grupos de mujeres donde puede estar un hombre, grupos donde suelen platicar sólo mujeres o exclusivamente hombres. Es común encontrar reuniones en las áreas de esparcimiento (jardines, mesas de la cafetería, pasillos), realizando diversas actividades, entre ellas jugar cartas a media mañana. Este es un entretenimiento preferentemente masculino, según comenta una estudiante:

Las cartas o las que juegan de yu-gi-oh pues no, ni le entiendo, luego cada cosa que dicen los chicos de combinaciones de monstruos [risas], ahí que se hagan pelotas ellos y pues para ¿deportes? como que siento que no tengo tiempo, pero si me gustaría jugar básquetbol (Dulce, estudiante de matemáticas)

Además de esta opinión, es posible que el juego de cartas implique apostar dinero (ganar o perder) y, como otro tipo de entretenimientos de hombres, supone la oportunidad de demostrar hombría y poder, acto que corresponde a rituales de la masculinidad hegemónica. En el escenario universitario estudiado, las pláticas en voz alta y las conversaciones bulliciosas, combinadas con el uso de palabras altisonantes y ofensivas, son también formas de expresión masculina que tienen lugar en la cafetería, en el cubículo de fotocopiado o en las canchas deportivas.

Por otra parte, la negación de las mujeres a participar en este tipo de prácticas de esparcimiento tiene como respuesta el desagrado, desconocimiento del juego o no comprenderlo. En concreto, no es de su interés o, como lo señala Dulce, no lo practican por falta de tiempo para aprender y practicar alguna otra actividad deportiva. Es oportuno decir que la difusión de las prácticas 
físicas no logra efectividad, pues «casi no hay mujeres que participen en el deporte», «no jalan para eso», «no a todas les llama la atención lo mismo». Pero la razón principal, según indicaron, es porque no logran reunir el número de integrantes para formar un equipo y por ello se forman grupos mixtos. Las estudiantes permanecen dentro del salón de clase leyendo, haciendo algún trabajo escolar, comiendo o platicando con amigas. En ocasiones, cuando salen del aula, acompañan a los hombres a sus actividades, aunque la mayoría de veces se mantienen como espectadoras.

En concreto, las mujeres muestran mayor interés por los asuntos académicos que las actividades deportivas, mientras que los hombres prefieren practicar fútbol o básquetbol. La cuestión es cómo se espera que las mujeres participen de forma activa en la vida deportiva si no se generan las instancias para hacerlo. La separación de los espacios físicos que ocurre desde la escuela inicial en cuanto a las oportunidades para estimular el deporte continúa en la universidad.

Al seguir explorando, se cuestionó ¿cómo te tratan tus compañeros? Un comentario unísono hizo escuchar un «nos llevamos bien, hay respeto». Sin embargo, relatos más precisos de las estudiantes parecen contradecir esta opinión fuertemente generalizada.

Yo evito hablar con mis compañeros por ciertas razones que se han dado, ya no me siento tan a gusto. Hablan de las chicas del salón, de quién es virgen y quién no (Dulce, estudiante de matemáticas)

Siempre he sido un poco reservada, siempre guardo mi distancia. Me llevo bien con ellos pero hasta cierto punto. Pues los chicos de repente sacan temas que incomodan, de qué tan buena está una chica, pero es buena la relación (Marly, estudiante de matemáticas)

Anteriormente tenía más amigos hombres, pero ahora con ellos no me llevo bien, no me siento en confianza (Mony, estudiante de química industrial)

A veces es como regular, porque unos como hacen sus grupos unos se apoyan y otros no (Roberta, estudiante de mecánica))

De esta manera, la opinión masculina de «sin ellas no sería lo mismo» se convierte en un mero discurso que no corresponde con las acciones. Las voces de las estudiantes indican la necesidad de evitar la conversación con los hombres y marcar su distancia, es decir, desconfiar de los compañeros por temor a que rebasen los límites del respeto. Estos límites se establecen para evitar la transgresión de cuestiones regularmente asociadas a la moral de las jóvenes o a su sexualidad, pero además para evadir las actitudes groseras o indecorosas que ellos manifiestan y que las coloca en una posición de poca confianza y expuesta a recibir ofensas o actitudes de irrespeto. 
Frente a estas experiencias, una estudiante opinó que entre mujeres suelen llevarse mejor y al parecer mantienen relaciones más armónicas y amables: «El grupo no es muy unido, las seis chicas que estamos aquí nos llevamos muy bien, con los muchachos no hemos tenido esa compatibilidad» (Nora, estudiante de mecánica).

Otra forma de socialización sexista transita por el lenguaje corporal y se manifiesta en posturas físicas, gestos faciales y articulación de manos para emitir señas. Algunas de las estudiantes manifestaron incomodidad al sentirse observadas de forma lasciva, porque les hacían guiños o algún otro tipo de movimiento inconveniente (señas obscenas, movimiento de labios). Incluso, en algún caso, se narró una actitud arrogante que denotó prepotencia o menosprecio hacia el resto: «Sí, de repente un compañero tiene una actitud de 'Yo lo sé todo y Ustedes no' son soberbios» (Marly, estudiante de matemáticas). Según Marly, esos comportamientos son más agudos cuando hay una mayoría de mujeres.

En otras narraciones, las estudiantes comentaron que algún compañero puede actuar de esa forma, pero «sin un afán de molestar». Esta afirmación concuerda con una manifestación de la ceguera de género que es un padecimiento normalizado que hace que la gente (mujeres y hombres) no distinga las desigualdades y los actos de discriminación en razón del género, y sienta que no existen tales actos o incluso asuman una posición cobijada por el discurso del sexismo benevolente. Es decir, esas actitudes que consideran de forma estereotipada y limitada a ciertos roles y que se manifiestan en un tono afectivo y positivo.

\subsection{Sociabilidad y convivencia con el profesorado: «me ignora»}

El aula en la universidad es uno de los espacios de interacción donde se continúa generando la reproducción cultural, social y de género, iniciada en el núcleo familiar. El salón de clases es el sitio donde se provee, distribuye y beneficia a ciertos agentes. Las evaluaciones que el profesorado realiza en el aula acreditan o desacreditan al aprendizaje adquirido, al mismo tiempo que se convierten en herramientas de negociación y reconocimiento, lo que puede producir desigualdad de oportunidades. Con la intención de indagar quién tiende a presentar trabajos escolares de mayor relevancia, la respuesta fue que las mujeres, aunque ellas también se declaran como quienes piden mayor apoyo: «Las mujeres porque somos más responsables, los hombres como que no la hacen» (Dulce, estudiante de matemáticas).

Además, las entrevistadas aseguran que sus compañeros opinan que la presentación de los trabajos de ellas es mejor porque «son más detallistas y dedicadas, de hecho desde los apuntes son más ordenadas y le ponen colorcitos 
y uno [los hombres] nada más con color negro». Esta acción podría estar reflejando la necesidad de las mujeres de ser reconocidas intelectualmente, tener mayor disposición, dedicación y tiempo para las tareas escolares. Otras respuestas indicaron que la calidad de los trabajos depende de la pasión por la carrera, ello supone que las mujeres son más sensibles y amorosas, y por tanto estos atributos de género son idóneos para ciertas actividades en este campo.

En cuanto a la expresión sin censura de sus opiniones, la mayoría dijo sentir libertad para hacerlo, pero muchas manifestaron inseguridad, temor a equivocarse o a recibir críticas:

Si bueno tengo la idea pero de eso a expresarla, aun no tengo esa seguridad en mí (Dulce, estudiante de matemáticas)

Tengo miedo a equivocarme y que los demás se burlen (Ana, estudiante de química)

A veces sí y a veces no, sólo con personas que me llevo sí doy mi opinión sino siento miedo al rechazo, timidez (Roberta, estudiante de mecánica)

A lo mejor no tienes clara la idea y dices 'si digo', mejor no digo [risas] y mejor te quedas callada, por miedo mejor te quedas callada, es la inseguridad de tu respuesta, quieres decir esto pero dices 'y isi no estoy bien?' mejor no digo nada, regreso a lo mismo de antes (Silvia, estudiante de sistemas electrónicos)

Frente al temor a expresar sus opiniones o saberes, y para no ser objeto de burlas, las mujeres prefieren no participar, evitarlo, retraerse, aislarse o guardar silencio. En conjunto, estas acciones denotan las estrategias de resguardo/ protección que adoptan en contextos de alta concentración masculina donde al parecer se han acostumbrado a transitar no sólo como seres humanos invisibles o mimetizadas, sino además con voces silenciadas. En opinión de las estudiantes, existe una marcada predilección hacia los hombres que se muestra en mayor atención:

En algunas ocasiones los profesores dan preferencia a compañeros que empiezan a destacar más, 'a él, a él, a él', y a los demás los olvida, o en algunos casos dan preferencia a los que vienen más arreglados (Roberta, estudiante de mecánica)

Al respecto, algunos testimonios ratifican situaciones de desigualdad (de género y de clase) que se vivencian en el aula entre el profesorado y el estudiantado: «Estas pensando en la respuesta y el profesor interrumpe y dice: ‘Espera! que tu compañero responda'...» (Roberta, estudiante de mecánica); «Sí, yo si me he sentido agredida en esa forma, a veces tomamos una clase en la que tengo dudas y pregunto, y me ignora, y pues no sé si hablé muy bajito» 
(Vero, estudiante en sistemas electrónicos); «Con los hombres se lleva más que con nosotras, a nosotras no nos pela» (Tere, estudiante en química industrial).

Las mujeres atribuyen y justifican el hecho de que el profesorado no les ceda la palabra, se la otorgue a otros (lo que refuerza el dominio conversacional masculino) e ignore su presencia se debe al tono y al volumen de la voz o a la empatía que entre varones puede generarse. En ninguno de los casos las estudiantes objetaron, alzaron el tono de la voz o hicieron alguna otra acción asertiva para afirmar su presencia y reclamar sus derechos.

En el disimulo de enseñar se disfrazan imposiciones, ejercicio de autoridad y poder, y hasta distanciamiento entre el profesorado y el estudiantado, particularmente con las estudiantes. Si bien las respuestas indican que para algunas la relación con el profesorado tiende a ser buena, también se puede observar que para otras en algunas ocasiones es poco accesible y sexista:

Si una mujer había hecho bien el ejercicio y un hombre también, primero lo pasaba a él, los hombres son así, si diferencian un poco (Vero, estudiante de sistemas electrónicos)

Regular, siento que es por no me acerco mucho a ellos porque si les preguntas algo te van a regañar o te van a llamar la atención de que no pones atención en la clase (Roberta, estudiante de mecánica)

No te conceden platicar o decir tus razones (Manuela, estudiante de química)

De esta manera, la práctica docente se enmarca en una relación de supremacía que sostiene roles tradicionales y los reproduce, donde el enseñar es un círculo cerrado que no admite dudas en el aprendizaje. En esta dinámica está prohibido resolver dudas y «acercarse para reclamar», especialmente las mujeres, pues el saber docente es adoptado como el bastión de poder masculino. No obstante, se debe señalar que algunos profesores intentan incluir a las mujeres en un mundo académico, en el que parece ellas llegaron tarde: «Los profesores han dicho 'que debemos razonar como matemáticos' [risas] y entonces dije, 'yo tengo que hacer eso'...» (Dulce, estudiante de matemáticas).

Finalmente, si bien parece existir una norma no escrita como expresión de curriculum oculto de género que enuncia que «en este espacio no hay compañeras que le crucen la pierna al profesor», algunos testimonios aseguran conocer el caso de profesores que «piden favores sexuales a las universitarias» y otros que de manera pública manifiestan actitudes ofensivas y sexistas:

Yo sí, porque hay un profesor que habla mucho sobre las mujeres, muy incómodo, muy morboso o sea no específica hacia nosotras, pero yo creo que sí, muy grotesco, por respeto a nosotras no debería hablar así, que lo haga con los hombres (Sara, estudiante de mecánica) 
Guardar silencio, permanecer calladas y no denunciar son enseñanzas aprendidas por las estudiantes. Todas estas prácticas se han forjado desde sus contextos familiares y reforzadas en un sistema educativo patriarcal que reproduce situaciones donde la socialización diferenciada de género y las prácticas de sociabilidad y convivencia están fuertemente enraizadas en los pensamientos y actuares de la masculinidad hegemónica.

\section{A MODO DE REFLEXIÓN}

Las aulas universitarias mixtas son una realidad. Sin embargo aún a mitad de la segunda década del siglo XXI, estudiar alguna carrera en un escenario fuertemente masculinizado es tener un arma de doble filo. Para las participantes en esta investigación, los desafíos se perfilan en tres momentos: al decidir ingresar, al transitar en la formación y durante las expectativas laborales. En todas las circunstancias (entrar, permanecer y ser reconocidas en el campo laboral), el dogma masculino las obliga a demostrar continuamente sus aptitudes y habilidades.

Las mujeres que estudian alguna de las seis ingenierías de la FCBIyT han ingresado para probar sus capacidades y desarrollarse intelectualmente, a pesar de que la vía de acceso no es nada sencilla. La opción es consciente, saben que las disciplinas son áreas de gran dificultad y que demandan alta concentración e inteligencia, atributos que ellas poseen. Sólo una de las diecisiete entrevistadas dijo haber querido estudiar una profesión del área de las ciencias sociales. Esta información indica que las estudiantes cursan la ingeniería que eligieron por convicción, deseo y capacidad intelectual. Pero reconocen que la permanencia no es fácil y su recorrido no es libre, persistir las enfrentará con una montaña a escalar. Las opciones pueden ser «pasar de largo» como una actitud voluntaria para evadir situaciones incomodas, pasar desapercibidas, camufladas con vestimenta que las haga parecer igual al resto -la mayoría varones-, utilizar escaso o nulo maquillaje, norma que puede ser permisible cuando ellas participen de modo oficial en actos públicos e incluso transitar de modo invisible.

Los hombres, por el contrario, sin ningún problema ocupan confortablemente los espacios en esta facultad, juegan, hablan en voz fuerte y con palabras altisonantes. Ellos actúan así porque están en ambientes reconocidos, son de ellos, ellos son los dueños, los propietarios. Las mujeres que eventual y transitoriamente son visitantes o huéspedes, escasamente se dejan ver, apenas se escuchan sus voces. Ellas prefieren arrinconarse, estar juntas, platicar, realizar actividades académicas, hacer uso de la biblioteca o de los laboratorios, pues exponerse las coloca en posición de vulnerabilidad y en «la mira», en el centro 
o el blanco de la atención que a veces puede ser bastante benevolente y otras, la mayoría, punzante y sexista.

Si bien, en la generalidad, las mujeres no distinguen de modo directo algún tipo de malos tratos por parte de los hombres, algunas otras sí lo perciben y otras más justifican el actuar masculino. Recordemos que en estas áreas, la formación pro igualdad es aún una historia no iniciada. Sin embargo, hay ciertos asuntos a reflexionar: los hombres hablan de ellas, de su sexualidad y de sus cuerpos. Ellas son identificadas como objetos que pueden lastimar hablando mal, desprestigiando, haciendo correr rumores sobre su vida íntima. Por su parte, las mujeres de manera inmediata no reaccionan frente a prácticas sexistas, ellas estudian, son disciplinadas, se resguardan, comparten con otras y disfrutan su permanencia en la Universidad.

Para las estudiantes es preferible no mantener contacto o evitar confrontaciones por temor a exponerse y ser la siguiente en la lista de los murmullos malsanos y crueles. Estar junto a ellos no es un buen sitio, se corre riesgos y se tienen que tolerar conversaciones misóginas y denigrantes. Es mejor estar juntas, entre mujeres, hablar de temas que importan, hacer tareas escolares, apoyarse, sentirse bien. Ellas son más responsables, cumplen más, son ordenadas, detallistas, inteligentes, pulcras, atributos que denotan estereotipos de género, pero que utilizan a su favor. Sin embargo, a pesar de todas estas cualidades, no logran solidificar prácticas de asertividad como una habilidad personal que les permita expresar directamente los propios sentimientos, opiniones y pensamientos, y defender sus derechos en el momento oportuno, de la forma adecuada.

A pesar de estas virtudes femeninas, las estudiantes no sienten seguridad para manifestar sus opiniones en el aula. La mayoría permanece callada, no habla, no discute. El temor a equivocarse es uno de los menores, el no sentirse apoyadas por el profesorado que las censura, no les da oportunidad, las ignora o anula sus opiniones, es más fuerte que sus ideas. En contraparte, surge el temor a que no les den su lugar, no les prestan tiempo, no toman en cuenta sus situaciones, son morbosos, se presume piden favores sexuales, las hostigan y no les guardan respeto.

En su futuro próximo distinguen un lugar en la vida laboral cargando a cuestas el dominio del espacio doméstico como exclusivo de ellas, el cual sostiene además la creencia social -de ofertantes de empleo y de la sociedad en conjunto-que estas áreas son para los hombres. El mañana no es alentador para la formación científica, ellas se retratan permaneciendo en «rincones»o encerradas (laboratorios, actividades gerenciales, empresas), de tal forma que la investigación se vuelve un espacio privilegiado y otras áreas de innovación 
tecnológica y apertura laboral se diseminan, y la docencia es cada vez más un empleo precario.

A pesar de todas las dificultades, algunas lo han hecho, lo continúan haciendo y han aprendido a sortear obstáculos y zanjear las brechas de forma tal que el camino andado sea más plano y menos enlosado, y favorezca la incursión de generaciones venideras. La Universidad Autónoma de Tlaxcala, en este gran reto, debe emprender acciones normativas y prácticas para que en este espacio de sociabilidad y convivencia entre géneros sea en un presente y en un futuro inmediato más justo e igualitario para quienes lo habitan.

\section{REFERENCIAS BIBLIOGRÁFICAS}

Álvarez-Gayou Jurgenson, Juan Luis. Cómo hacer investigación cualitativa. México: Paidós Educador, 2004.

Álvarez Liébana, Natalia, Ana Moreno, Vicente Riveira y Carlos Mataix. «Mujeres e Ingeniería. Caso de estudio en la ETSII-UPM». Ponencia presentada en $4^{\text {th }}$ International Conference on Industrial Engineering and Industrial Management. 9 de julio de 2016. Disponible en: http://www.anamorenoromero.net/documentos/Anexo4_ETSII-UPM.pdf

ANUIES. Anuarios estadísticos de Educación Superior. Disponible en: http://www. anuies.mx/iinformacion-y-servicios/informacion-estadistica-de-educacionsuperior/anuario-estadistico-de-educacion-superior, 10-12-2016.

Arango Gaviria, Luz Gabriela. «Género e ingeniería: la identidad profesional en discusión». Revista Colombiana de Antropología 42 (2006): 129-156.

Ballarín Domingo, Pilar. «Los códigos de género en la universidad». Revista Iberoamericana de Educación 68 (2015): 19-38.

Buquet, Ana, Jennifer Ann Cooper, Mingo, Araceli, Hortensia, Moreno. Intrusas en la universidad. México: UNAM, 2013.

Camacho, Gloria. Mujeres Fragmentadas. Quito: CEPLAES, 1997.

Cantillo Barrios, Ligia y Delma Rocha Álvarez. «Las relaciones de género y la educación superior en Colombia. Estudio de caso: Universidad del Atlántico», Estudios de género. Nuestros pasos en el Caribe colombiano. Comp. Rafaela Vos Obeso. Colombia: Universidad del Atlántico, 2007, 1-18.

Castro, Roberto y Verónica Vázquez García. «La Universidad como espacio de reproducción de la violencia de género. Un estudio de caso en la Universidad Autónoma Chapingo, México». Estudios Sociológicos 78 (2008): 587-616.

Coates, Jennifer. Mujeres, hombres y lenguaje. Un acercamiento sociolingüístico a las diferencias de género. México: Fondo de Cultura Económica, 2009.

Costa Ferreira, María Regina y Rogério Goulart Silva. «La cultura del patio de recreo: las relaciones de niños y. Niñas en primaria». Ponencia presentada en el XXVII Congreso de la Asociación Latinoamericana de Sociología. VIII Jornadas 
de Sociología de la Universidad de Buenos Aires (2009) 9 de enero de 2016. Disponible en: http://www.aacademica.org/000-062/823

Díaz Cruz Rodrigo. «La vivencia en circulación. Una introducción a la antropología de la experiencia». Alteridades 7 (1997): 5-15.

Flores Bernal, Raquel. «Violencia de género en la escuela: sus efectos en la identidad, en la autoestima y en el proyecto de vida». Revista Iberoamericana de Educación 38 (2005): 67-86.

Gallego Noche, Beatriz. «La igualdad de género en la práctica deportiva y su contribución a la educación para la ciudadanía y la democracia». Journal for Educators, Teachers and Trainers 5.3 (2014). 17 de julio de 2016. Disponible en: http://rodin.uca.es/xmlui/bitstream/handle/10498/16949/La\%20igualdad\%20 de\%20g\%C3\%A9nero\%20en\%20la\%20pr\%C3\%Alctica\%20deportiva_ solo\%20art\%C3\%ADculo.pdf? sequence $=1$

Gallego Noche, Beatriz y Araceli Estebaranz García. Mujeres que abren camino en el deporte: factores influyentes en las situaciones de igualdad y discriminación. Universidad de Sevilla. 14 de enero de 2016. Disponible en: http://www.mujerydeporte.org/documentos/docs/MUJERES\%20QUE\%20 ABREN\%20CAMINO\%20EN\%20EL\%20DEPORTE\%20FACTORES\%20 IN F LUYEN TES\% 20 EN \% 20 LAS\% 20SITUACIONES\% 20 DE \% 20 IGUALDAD\%20Y\%20DISCRIMINACI\%C3\%93N.pdf

García Pérez, Rafael, Estrella Ruiz Pinto y Ángeles Rebollo Catalán. «Preferencias relacionales de género en el contexto escolar: Una nueva medida para el diagnóstico de relaciones de género en educación». RELIEVE 1, (2016): 1-21.

Guevara Ruiseñor, Elsa y Alba García López. «Orden de género y trayectoria escolar en mujeres estudiantes de ciencias exactas y naturales». Investigación y Ciencia 46 (2010):10-17.

Hernández, Alfonso. «La violencia de género y sus prácticas en la Universidad de Guadalajara». Violencia de Género en Instituciones de Educación Superior en México Coord. Rosa María González Jiménez, México: IPN-CONACYT, 2013, 135-172.

Larena Fernández, Rosa y Silvia Molina Roldán. «Violencia de género en las universidades: investigaciones y medidas para prevenirla». Trabajo Social Global, Revista de Investigaciones en Intervención Social 2 (2010): 202-219. 3 de febrero de 2016. Disponible en http://tsghipatiaeditorial.com/index.php/tsg1

López Francés, Inmaculada, María Isabel Viana Orta y Sánchez Sánchez, Beatriz. «La equidad de género en el ámbito universitario: ¿un reto resuelto?». Revista Electrónica Interuniversitaria de Formación del Profesorado, 19.2 (2016): 349-361. 26 de abril de 2016. Disponible en http://dx.doi.org/10.6018/ reifop.19.2.211531

Lorente Acosta, Miguel. «Violencia de género, educación y socialización: acciones y reacciones». Revista de Educación 342 (2007): 19-35. 
Maceira Ochoa, Luz. «Investigación del currículo oculto en la educación superior: alternativa para superar en sexismo en la escuela». Revista de Estudios de Género. La Ventana 21 (2005): 187-227.

Malegarie, Jessica y Romina Tavernell. «Educación para la integración ¿Cómo se aborda la diversidad en el aula?». Question. Revista Especializada en Periodismo y comunicación 19 (2011): 62-124.

Martínez Lozano, Consuelo Patricia y Daniel Solís Domínguez. «El entorno escolar y familiar en la construcción de significaciones de género y sexualidad en jóvenes de Guadalajara». Revista de Estudios de Género. La Ventana 29 (2009): 146-183.

Martínez Moscoso, Dolores Marisa. Práctica docente con equidad de género. Una guía de trabajo. México: Universidad de Guadalajara, 2012.

Mejía Navarrete, Julio. «Problemas centrales del análisis de datos cualitativos». Revista Latinoamericana de Metodología de la Investigación Social 1 (2011): 47-60. 8 de marzo de 2015. Disponible en: http://www.relmis.com.ar/ojs/index. php/relmis/article/view/11/13

Mingo, Araceli y Hortensia Moreno. «El ocioso intento de tapar el sol con un dedo: violencia de género en la universidad». Perfiles Educativos 148 (2015): 138-155.

Mingo, Araceli. « ¿Ni con el pétalo de una rosa? Discriminación hacia las mujeres en la vida diaria de tres facultades». Revista Iberoamericana de Educación Superior 18 (2016): 24-41.

Oliveros Ruiz, María Amparo, Eduardo Cabrera Córdoba, Benjamín Valdez Salas y Michael Schorr Wienner. «La motivación de las mujeres por las carreras de ingeniería y tecnología». Entreciencias. Diálogos en la sociedad del conocimiento 9 (2016): 89-96.

Paulín, Horacio, Marini Tomasini, Paula Bertarelli, Florencia D’aloisio, Guido García Bastán, Soledad Martínez, Guadalupe Torres y Ailín Vallejo. «Sociabilidad juvenil en la escuela media: Análisis de las prácticas relacionales y los procesos de reconocimiento entre estudiantes». VII Jornadas de Sociología de la UNLP. (2012). 4 de diciembre de 2015. Disponible en: http://www.memoria.fahce. unlp.edu.ar/trab_eventos/ev.2174/ev.2174.pdf

Peña, María Alejandra y Lillo Buschiazzo. Elección de carreras universitarias y género el Caso de trabajo social e ingeniería. Tesis para optar al grado de magister en estudios de género y Cultura, mención ciencias sociales, Universidad de Chile. (2006). 8 de junio de 2016. Disponible en: http://www.ciegchile.com/ wp-content/uploads/2014/05/cs39-penaylillom222.pdf

Pérez Naranjo, Julia y Armando Martín Ibarra López. «La socialización Mediada en las Interacciones juveniles. La Construcción del Yo en Facebook». Revista de Tecnología y Sociedad 4 (2013): 1-12. 
Preciado Cortés, Florentina Karla Kae Kral y María Guadalupe Álvarez Ramón. «Navegando entre dos mares: mujeres en el contexto de la cultura de ingeniería». Revista Iberoamericana de Educación 68 (2015): 39-58.

Rebolledo, Loreto. «Género y Espacios de Sociabilidad. La Escuela como Lugar de Encuentro con los 'Otros'». II Congreso Chileno de Antropología. (1995): 89-97. 9 de junio de 2015. Disponible en: https://www.aacademica.org/ii.congreso. chileno.de.antropologia/16.pdf

Sánchez Silva, Modesto. «La metodología en la investigación cualitativa». Revista Mundo Siglo XXI 1 (2005):115-118. 6 de agosto de 2015. Disponible en: http://132.248.9.34/hevila/MundosigloXXI/2005/nol/8.pdf

Simmel, George. Sobre la individualidad y las formas sociales. Buenos Aires: Universidad Nacional de Quilmes, 2002.

Vázquez García, Verónica y María Eugenia Chávez Arellano. «Masculinidad hegemónica en la Universidad Autónoma Chapingo. Un estudio de caso entre estudiantes». Textual 49 (2007): 41-65.

Weiss, Eduardo. «Más allá de la socialización y de la sociabilidad: jóvenes y bachillerato en México». Educ. Pesqui 41 (2015): 1257-1272. 